\title{
Maintenance of adaptive differentiation by Wolbachia induced bidirectional cytoplasmic incompatibility: the importance of sib-mating and genetic systems
}

\author{
Antoine Branca*1,2, Fabrice Vavre ${ }^{3,4}$, Jean-François Silvain ${ }^{1,2}$ and \\ Stéphane Dupas ${ }^{1,2,5}$
}

Address: ${ }^{1}$ Unité de Recherche IRD 072, CNRS UPR9034, Laboratoire Evolution, Génome et Spéciation, Gif-sur-Yvette, France, ${ }^{2}$ Université ParisSud 11, Orsay, France, ${ }^{3}$ CNRS UMR5558, Laboratoire de Biométrie et Biologie Evolutive, Villeurbanne, France, ${ }^{4}$ Université Lyon 1, Villeurbanne, France and ${ }^{5}$ Unité de Recherche IRD 072, Pontificia Universidad Católica del Ecuador, Quito, Ecuador

Email: Antoine Branca* - Antoine.Branca@legs.cnrs-gif.fr; Fabrice Vavre - vavre@biomserv.univ-lyon1.fr; Jean-François Silvain - JeanFrancois.Silvain@legs.cnrs-gif.fr; Stéphane Dupas - Stephane.Dupas@legs.cnrs-gif.fr

* Corresponding author

Published: 4 August 2009

BMC Evolutionary Biology 2009, 9:185 doi:10.1 |86/147|-2148-9-185
Received: 26 November 2008

Accepted: 4 August 2009

This article is available from: http://www.biomedcentral.com//47/-2/48/9//85

(c) 2009 Branca et al; licensee BioMed Central Ltd.

This is an Open Access article distributed under the terms of the Creative Commons Attribution License (http://creativecommons.org/licenses/by/2.0), which permits unrestricted use, distribution, and reproduction in any medium, provided the original work is properly cited.

\begin{abstract}
Background: Bacteria of the genus Wolbachia are reproductive parasites widespread among arthropods. The most common effect arising from the presence of Wolbachia in a population is Cytoplasmic Incompatibility $(\mathrm{Cl})$, whereby postmating reproductive isolation occurs in crosses between an infected male and an uninfected female, or when a male is infected with a different strain of Wolbachia to that of the female (bidirectional $\mathrm{Cl}$ ). Previous theoretical models have demonstrated that bidirectional $\mathrm{Cl}$ can contribute to the genetic divergence of populations in haploid and diploid organisms. However, haplodiploid organisms were not considered in these models even though they include Nasonia parasitoid wasps - the best example of the implication of Wolbachia in ongoing speciation. Moreover, previous work did not investigate inbreeding mating systems, which are frequently observed in arthropod species.

Results: We developed a stochastic two-island model which simulated three genetic scenarios, diploidy, haploidy, and haplodiploidy, with two $\mathrm{Cl}$ phenotypes being considered for the latter: (I) male development of female progeny; and (2) mortality of fertilized eggs. We also investigated the effect of varying the proportion of sib mating. In the model each allopatric population was initially fixed for a single allele at a nuclear locus under positive selection and infected with one strain of Wolbachia. Each simulation presupposed that the two populations were fixed for a different allele and a different strain of Wolbachia. The degree of genetic differentiation observed in the locus under selection due to bidirectional $\mathrm{Cl}$ was much lower for the two haplodiploid phenotypes than for either diploids or haploids. Furthermore, we demonstrated that sib-mating may compensate for the lower efficiency of bidirectional $\mathrm{Cl}$ in haplodiploids by maintaining genetic divergence.

Conclusion: Our model suggests that maintenance of genetic differentiation facilitated by Wolbachia is more likely to occur in diploids and haploids than in haplodiploids. However, increasing the level of sibmating may compensate for the weak effect of bidirectional $\mathrm{Cl}$ in haplodiploids. Our work therefore gives a potential explanation for why the haplodiploid Nasonia species, which are infected with bidirectionally incompatible Wolbachia strains and undergo sib-mating, have differentiated genetically and maintained this differentiation without premating isolation.
\end{abstract}




\section{Background}

Insects are frequently infected with bacterial symbionts. Many of them such as Wolbachia, Cardinium or Spiroplasma manipulate the reproduction of their hosts for their own advantage using different effects such as: feminization of genetic males, increase of male mortality (male killing), thelytokous parthenogenesis induction (PI) and, most commonly, cytoplasmic incompatibility (CI) [1-9]. All of these reproductive parasites are transferred vertically from mother to progeny via the cytoplasm of the eggs.

Unidirectional CI may occur when an uninfected female mates with an infected male $[2,10]$. The infected male's sperm is modified by the bacterial infection and, as a result, cannot fertilize eggs from female unless they are rescued by the same strain being present in the cytoplasm of the eggs $[11,12]$. In diploids, expression of CI results in no offspring development. In haplodiploids, the two CI phenotypes female egg mortality (FM) and male development of female eggs (MD), result in a male biased sex-ratio in the offspring [13-15]. In the FM phenotype, a reduction in the number of progeny is observed because only unfertilized eggs can develop into males (fertilized eggs being developmentally lethal) $[13,14]$. In the MD phenotype, the number of progeny is not affected in incompatible matings as both fertilized and unfertilized eggs develop into males due to the complete haploidization of fertilized eggs [15]. In diploid and haplodiploid genetic systems, the symbiont increases in frequency in the insect population by allowing infected females to produce more daughters than uninfected females increasing the reproductive success of infected females [14,16].

In addition to unidirectional CI, bidirectional CI may occur when the parents are infected by different strains of bacteria [15]. By generating such bidirectional reproductive incompatibilities, it has been suggested that CI promotes speciation [2,17-20]. Wolbachia is the most common of the endosymbionts causing $\mathrm{CI}$, and has recently been estimated to infect approximately $66 \%$ of all insect species, with prevalence rates within species ranging between 10-90\% [21]. The clearest example of Wolbachia inducing diversification is described in the three parasitic wasps of Nasonia species. In this haplodiploid insect, there is no premating isolation, but postmating isolation occurs as a result of bidirectional CI [22,23]. It is therefore suggested that Wolbachia bacteria are the main agent preventing mixing between Nasonia species. However a direct role of Wolbachia in speciation remains a controversial topic [24,25]. For example, a study of isofemale lines from natural populations of Drosophila simulans infected with different bidirectionally incompatible Wolbachia strains, showed no association between Wolbachia strain and genetic divergence at neutral nuclear markers, and no evidence of assortative mating behavior [26]. This case suggests that CI alone cannot induce speciation in Drosophila.
In contrast, another study of two Drosophila species showed that unidirectional CI leads to behavioral recognition and subsequent avoidance of the Wolbachia infected species by the uninfected one, hence maintaining premating isolation between species [27].

In an effort to investigate the possibility of CI promoting speciation, several theoretical models have been developed which focus on the impact of CI on genetic divergence. For example, theoretical frameworks showed that CI may strengthen genetic divergence between populations $[28,29]$ and, moreover, in some situations, bidirectional CI selects for premating isolation and could lead to speciation [30]. However, previous models on the effect of CI on genetic differentiation [28-31] did not consider some important points. (i) The genetic system of haplodiploid parasitoids differs markedly to that of haploids and diploids, yet the clearest evidence of the Wolbachia role in early speciation events is cited as being in the haplodiploid Nasonia species complex [22]. (ii) The MD and FM phenotypes common to haplodiploids may have different effects on genetic differentiation [14,32]. (iii) In parasitoids, selection acts preferentially on females because ecological niches are generally defined by the female choice of host [33], and by the female injecting into the host many of the factors required for development, including venom, ovarian proteins [34] and polydnaviruses [35]. (iv) Inbreeding may play a role in enhancing genetic differentiation among populations; near complete outbreeding, sib-mating, and parthenogenesis can all be observed within haplodiploid parasitoid species [36-38]. Furthermore, it has been previously demonstrated that sib-mating modifies the invasion dynamics of the Wolbachia infection [39,40] Finally, (v) Using a stochastic approach allows a finite population to be defined and enables the incorporation of genetic drift and other random evolutionary effects. In particular, Wolbachia spread dynamics are represented differently under a stochastic modeling simulation [41].

In this paper, we used a model similar to that of Telschow et al. [28] to investigate how CI contributes to maintaining divergence between two populations which have undergone different selection regimes, but after which nuclear genes and Wolbachia can be exchanged (Figure 1). Our approach is the first to $(i)$ integrate different levels of ploidy, (ii) integrate different CI phenotypes, (iii) consider female only selection, (iv) allow sib-mating, and (v) use a stochastic model.

\section{Methods \\ The model}

As in previous models $[28,30]$, simulations started after the contact of two populations, each infected with a different Wolbachia strain (Figure 1). Each population is monitored at a single nuclear locus under positive selection in 
Ancestral population

Ancestral allele on locus under positive selection

\section{Divergence in different environments}

\section{Acquisition of Wolbachia strains}
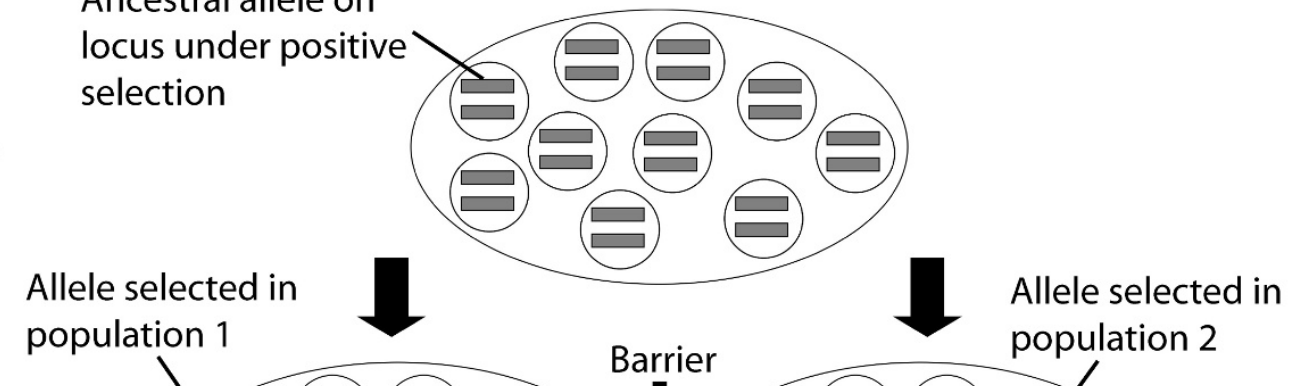
population 2
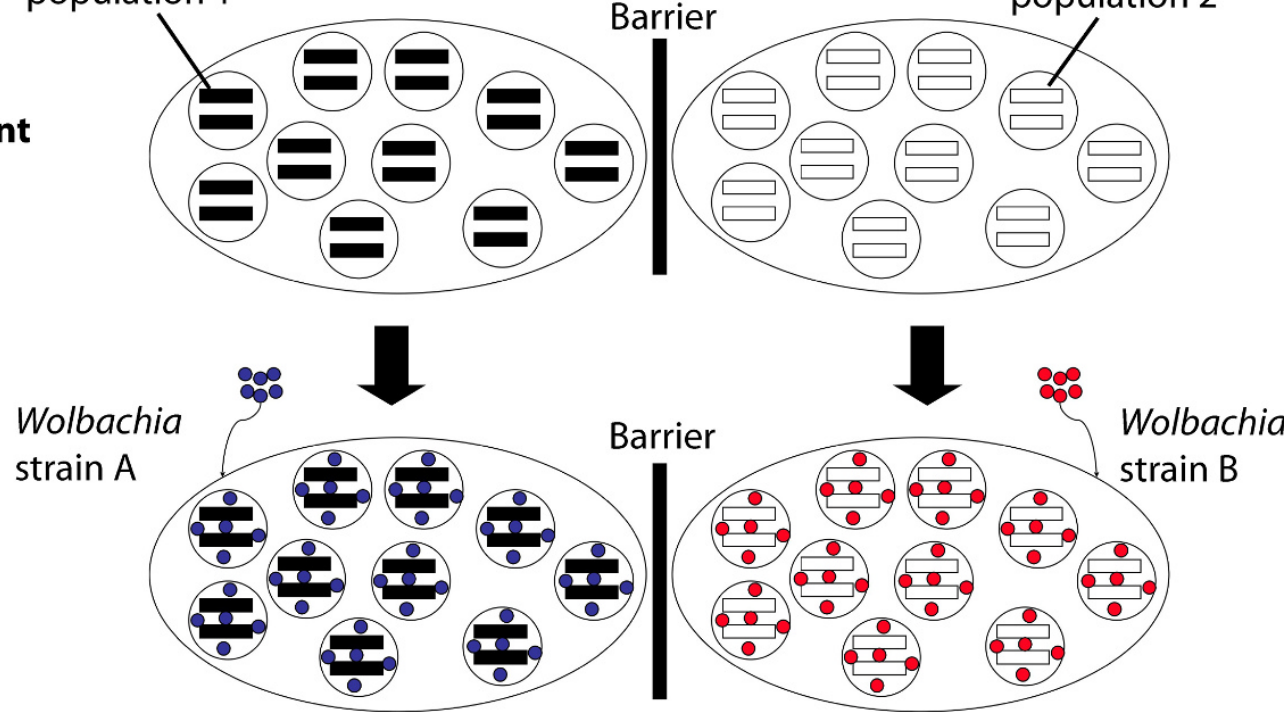

\section{Contact between populations (starting point of the simulations )}
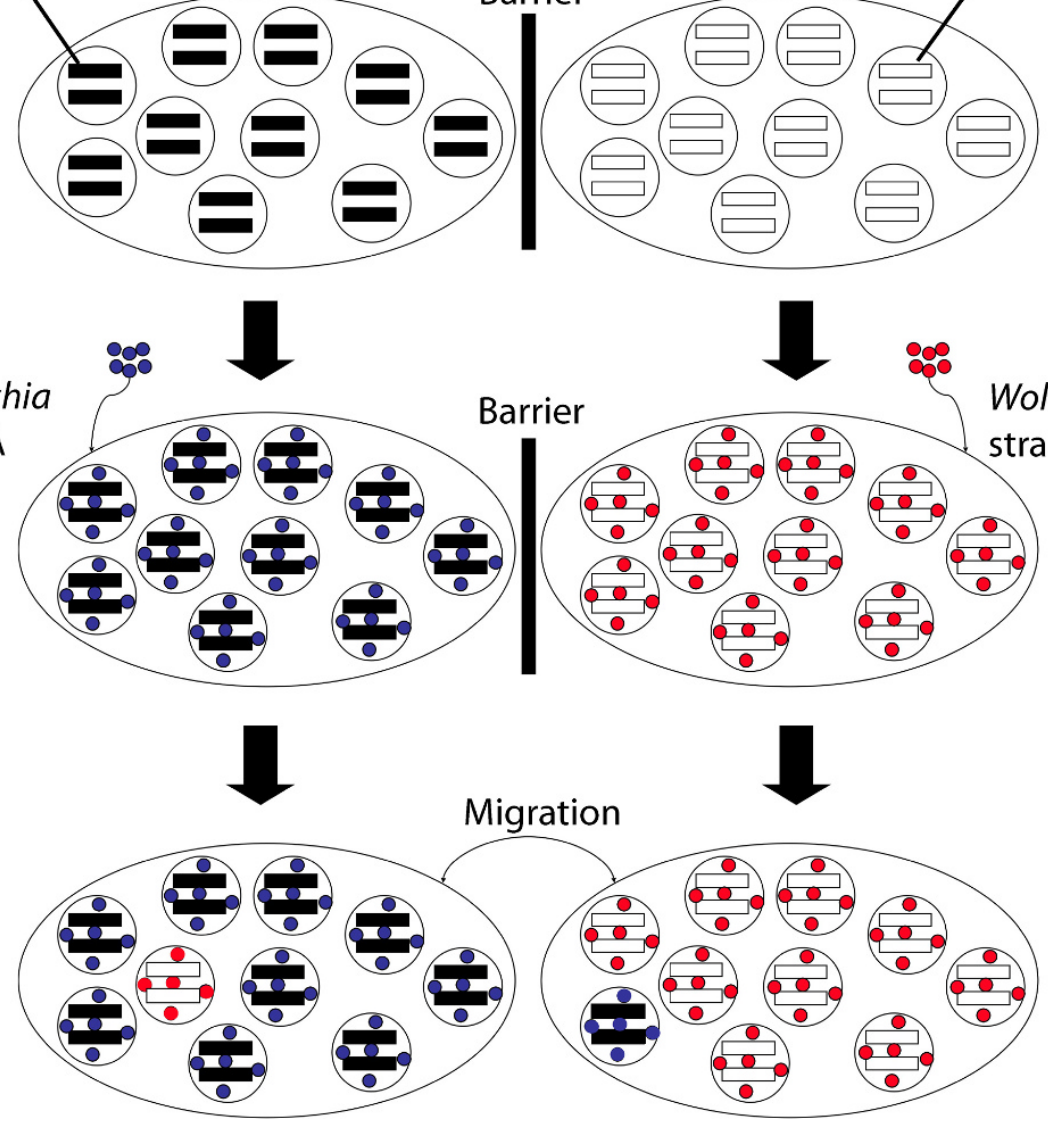

Migration
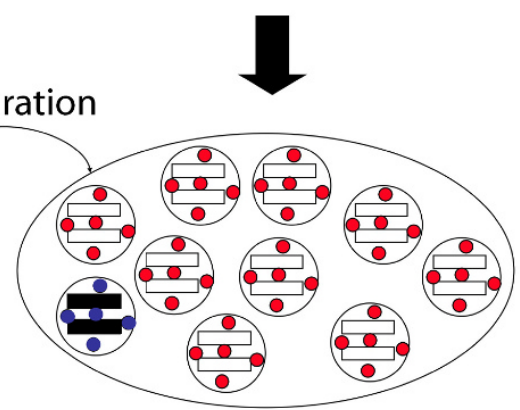

Figure I

Model scenario. This figure represents a schematic view of the evolutionary scenario implemented in the model for diploids. An ancestral population is first separated into two populations. Each population is adapted to its environment at a single nuclear locus which is under positive selection. After different strains of Wolbachia had invaded the two populations and reached saturation point, migration was permitted between the populations. Simulations started at this step.

females only, and each population is fixed for a different allele at this locus. We assumed a finite and constant population size $N$ for each generation $g$. Each individual, male or female, has a probability $m$ of migrating to the other population. Parameter $s_{i}$ is the selective coefficient, which is applied to a female possessing an allele not locally adapted to population $i$ on the selected nuclear locus.

The two populations each harbored a different Wolbachia strain, either $A$ or $B$, which were bidirectionally incompatible. We considered that infection by Wolbachia had no fit- ness effect. Each individual of the next generation had a probability $t$ of receiving Wolbachia from its mother. The probability of either bacterial strain $A$ or $B$ to express CI was $I_{A}$ and $I_{B}$ respectively.

Following Engelstädter et al. and Branca and Dupas $[39,40]$, we used a probability of each female mating with a brother. The proportion of female offspring, $P_{S R^{\prime}}$ was fixed to 1 male for 3 females, (e.g. for gregarious parasitoids [42]). Indeed, evolutionary stable sex-ratios are predicted to be female-biased when there is local mate 
competition [43]. Note however, that we did not see any significant effects of the primary sex ratio on either maintenance of Wolbachia diversity or the allelic frequency dynamics of the nuclear locus (results not shown).

Our model called CIParasitoid was developed using the " $\mathrm{R}$ " language and software R 2.8.0 [44]. The package includes helps and can be installed using standard R commands (see Additional files 1, 2, 3 and 4).

\section{Selection modeling}

In the two populations, females were fixed for a different allele on a single selected nuclear locus $V$. Typically this situation could represent the specialization of a parasitoid wasp (such as Nasonia) to different insect hosts. Thus, the locus $V$ governed the capacity to successfully parasitize a host. This locus bore two alleles $V_{1}$ specialized on host 1 and $V_{2}$ specialized on host 2 . We assumed semi-dominance of the alleles on locus $V$ meaning that heterozygotes randomly expressed one of the two alleles. Consequently, in the haplodiploid genetic system, for $i \neq j$ the probabilities of successful development of the progeny in the host $i$ were $1, \frac{s_{i}}{2}$ and $1-s_{i}$ for the homozygotes $V_{\mathrm{i}} V_{\mathrm{i}}$, heterozygotes $V_{\mathrm{i}} V_{\mathrm{j}}$ and homozygotes $V_{j} V_{j}$ respectively. In the haploid case, these probabilities were 1 and $1-s_{i}$ for the genotypes $V_{\mathrm{i}}$ and $V_{j}$ respectively. In the algorithm, the reproductive females were therefore sampled according to their genotypes on the locus $V$ while each male had the same probability of being sample, independently of its genotype.

As a result, we can calculate $F^{\prime}$, the expected number of reproductive females of each genotype after the selection process in haplodiploids and diploids for population $i$, as follows:

$$
\begin{aligned}
& E\left(F_{V i V i}^{\prime}\right)=\frac{F_{V i V i}}{F_{V i V i}+\left(1-\frac{s_{i}}{2}\right) F_{V i V j}+\left(1-s_{i}\right) F_{V j V j}} \\
& E\left(F_{V i V j}^{\prime}\right)=\frac{\left(1-\frac{s_{i}}{2}\right) F_{V i V j}}{F_{V i V i}+\left(1-\frac{s_{i}}{2}\right) F_{V i V j}+\left(1-s_{i}\right) F_{V j V j}} \\
& E\left(F_{V j V j}{ }^{\prime}\right)=\frac{\left(1-s_{i}\right) F_{V j V j}}{F_{V i V i}+\left(1-\frac{s_{i}}{2}\right) F_{V i V j}+\left(1-s_{i}\right) F_{V j V j}}
\end{aligned}
$$

And in haploids, as:

$$
\begin{aligned}
& E\left(F_{V i}{ }^{\prime}\right)=\frac{F_{V i}}{F_{V i}+\left(1-s_{i}\right) F_{V j}} \\
& E\left(F_{V j}{ }^{\prime}\right)=\frac{\left(1-s_{i}\right) F_{V j}}{F_{V i}+\left(1-s_{i}\right) F_{V j}}
\end{aligned}
$$

After this selection process, the program recorded all the reproductive individuals.

\section{Cytoplasmic Incompatibility modeling}

An incompatible cross occurred when an infected male mated with a female which was either uninfected or infected with a different bacterial strain.

In diploids and haploids, when CI occurred no offspring were produced. For haplodiploids, the CI effect was assumed to either result in all MD or all FM phenotypes. Consequently, for the MD phenotype, CI resulted in the development of only male progeny; for the FM phenotype, female progeny resulting from incompatible crosses did not developed and the number of progeny therefore decreased. To account for this, the probability of producing no offspring was equal to the primary sex ratio, $P_{S R^{\prime}}$ which was used as the probability of producing a female.

\section{Estimating genetic divergence and Wolbachia diversity maintenance}

Previous studies have measured genetic divergence induced by bidirectional CI on a selected locus by quantifying the reduction in the effective migration rate, or the production of hybrid offspring [28]. In the haplodiploid model, this variable is not easily estimated because CI does not result in the death of offspring but rather in the production of males. Therefore, this caused complications in the calculation of the reduction of the effective migration rate. Consequently, the effect of CI on genetic divergence was estimated by calculating a Fst on the selected nuclear locus as follows:

$$
F s t=1-2 \frac{p_{1} q_{1}+p_{2} q_{2}}{\left(p_{1}+p_{2}\right) *\left(q_{1}+q_{2}\right)}
$$

where $p_{i}$ and $q_{i}$ are the frequencies of allele 1 and 2 respectively in population $i$.

The maintenance of Wolbachia diversity, namely $W$, was measured by the probability of maintaining each Wolbachia strain in its respective population at a frequency above an arbitrary threshold of $75 \%$. 


\section{Default parameters of the simulations}

By default, the population size was set to $N=50$ individuals in each population, allowing for a high drift effect in the system, with simulations stopping after $g=100$ generations. Nevertheless, higher population sizes and longer running time were tested. The default values of parameters were set to $m=0.5, t=1, s_{i}=0.5$ and $=0$. In all simulations, we set $s_{1}=s_{2}$. Four different situations were calculated for bidirectional CI ranging from no CI to high levels of CI: $\left(I_{A}=0 ; I_{B}=0\right),\left(I_{A}=0.5 ; I_{B}=0.75\right),\left(I_{A}=0.75 ; I_{B}=\right.$ $0.75)$ and $\left(I_{A}=1 ; I_{B}=0.75\right)$. To test the significance of bidirectional $\mathrm{CI}$ on genetic divergence, we used a non-parametric unilateral Mann-Whitney test on Fst values. The $\mathrm{H}_{0}$ tested was "Fst in the bidirectional CI case was equal to Fst in the case where $I_{A}=0$ and $I_{B}=0 "$. The $H_{1}$ tested was "Fst in the bidirectional CI case was superior to Fst in the case where $I_{A}=0$ and $I_{B}=0$ ". This latter case was equivalent to a case without Wolbachia (i.e. Wolbachia therefore behaved as mitochondria). Fst and $W$ were calculated after 1000 repetitions.

\section{Results}

Differentiation at the selected nuclear locus due to bidirectional $\mathrm{Cl}$ in the different genetic systems (Table I; Figure 2; Figure 3)

Firstly, our results showed that $\mathrm{W}$ reached zero beyond a certain migration rate. It means that one Wolbachia strain has succeeded in saturating both populations (Figure 2). The previous model of Telschow et al. named this migration rate the threshold migration rate [28]. Threshold migration rates were lower in our stochastic model than in the previous deterministic model (Figure 3). However the threshold became closer at higher selection coefficients. Indeed, the threshold value was the same for both models where $\mathrm{si}=1$.
Secondly, for all genetic systems, we observed that Fst reached significantly higher values when bidirectional CI occurred than in situations with selection only (Table 1 and Figure 2). Bidirectional CI is therefore a force that increased genetic divergence at a selected locus.

Thirdly, by comparing the different genetic systems, we observed that threshold migration rates for $W$ were lower for haplodiploids than for diploids and haploids (e.g Figure $2 \mathrm{a}$ for $I_{A}=1$ and $I_{B}=0.75$, between 0.1 and 0.125 for haplodiploids and between 0.150 and 0.175 for haploids and diploids). In the same manner, lower Fst values were reached in the two haplodiploid phenotypes than in haploids and diploids. Indeed, in the three sets of incompatibility rates, $I_{A}$ and $I_{B}$ (Figure 2), Fst values in haplodiploids were never significantly higher than values in diploid and haploids (Mann-Whitney p < 0.001). In conclusion, when both Wolbachia strains were maintained, enhancement of genetic divergence by bidirectional CI was weaker in haplodiploids than in other genetic systems.

\section{Influence of selection on divergence under bidirectional $\mathrm{Cl}$ (Table 2; Figure 4)}

Firstly, in qualitative terms, in all genetic systems, W increased as the selection coefficient increased (Figure 4). Also, there was more effect of bidirectional CI on Fst as the selection coefficient increased.

Secondly, stronger selection was required to reach high values of $W$ for haplodiploids. Similarly, the effect of bidirectional CI on Fst in haplodiploids occurred for a narrower range of selection coefficient values than for other genetic systems (Table 2).

Thirdly, in diploids and haploids, for $I_{A}=0.75$ and $I_{B}=1$, enhancement of Fst by bidirectional CI was observed

Table I: Test of the impact of bidirectional $\mathrm{Cl}$ on genetic divergence for different values of $m$

\begin{tabular}{|c|c|c|c|c|c|c|c|c|c|}
\hline Migration rate & 0.2 & 0.175 & 0.15 & 0.125 & 0.1 & 0.075 & 0.05 & 0.025 & 0 \\
\hline & Diplo & Diplo & Diplo & Diplo & Diplo & Diplo & Diplo & Diplo & Diplo \\
\hline$I_{A}=0.75$ & & Haplo & Haplo & Haplo & Haplo & Haplo & Haplo & Haplo & Haplo \\
\hline \multirow[t]{3}{*}{$I_{B}=1$} & & & HDMD & HDFM & HDFM & HDFM & HDFM & HDFM & HDFM \\
\hline & & & & HDMD & HDMD & HDMD & HDMD & HDMD & HDMD \\
\hline & Diplo & Diplo & Diplo & Diplo & Diplo & Diplo & Diplo & Diplo & Diplo \\
\hline$I_{A}=0.75$ & & Haplo & Haplo & Haplo & Haplo & Haplo & Haplo & Haplo & Haplo \\
\hline \multirow{3}{*}{$I_{B}=0.75$} & & & HDMD & HDFM & HDFM & HDFM & HDFM & HDFM & HDFM \\
\hline & & & & HDMD & HDMD & HDMD & HDMD & HDMD & HDMD \\
\hline & & & & Diplo & Diplo & Diplo & Diplo & Diplo & Diplo \\
\hline$I_{A}=0.5$ & & & & & Haplo & Haplo & Haplo & Haplo & Haplo \\
\hline \multirow[t]{2}{*}{$I_{B}=0.75$} & & & & & HDFM & HDFM & HDFM & HDFM & HDFM \\
\hline & & & & & & HDMD & HDMD & HDMD & HDMD \\
\hline
\end{tabular}

Each box is filled with the name of the system where bidirectional $\mathrm{Cl}$ significantly increased the Fst value on the selected locus (Mann-Whitney test, $p$-value $=0.05$ ) . Diplo $=$ Diploids, Haplo $=$ Haploids, HDFM $=$ Haplodiploid Female Mortality and HDMD $=$ Haplodiploid Male Development. Fixed parameters were set to $s_{i}=0.5, P_{S R}=0.75,=0, t=1, g=100, N=50$. 


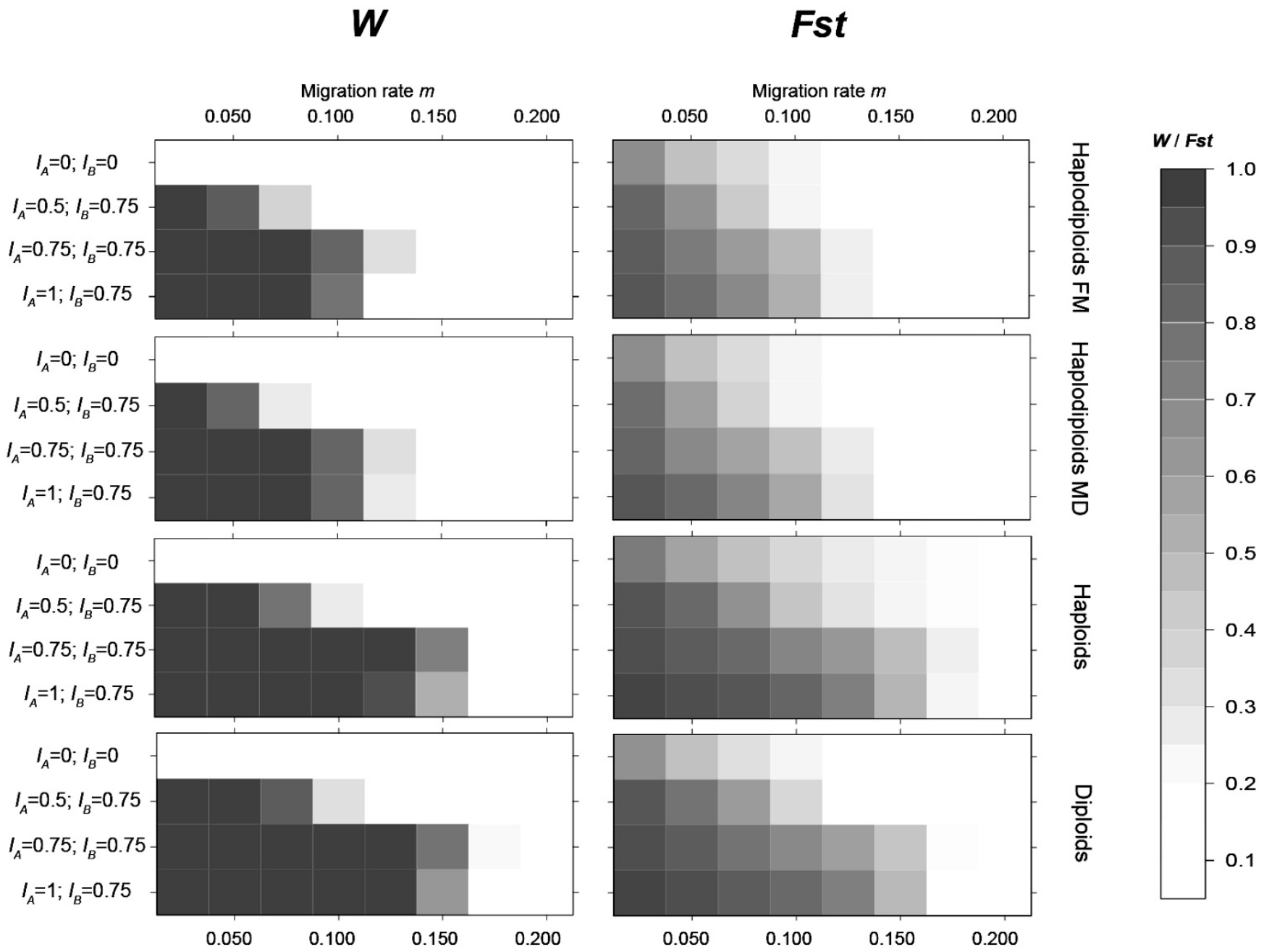

Figure 2

Effect of migration rate $\boldsymbol{m}$ on idirectional $\mathbf{C l}$ and genetic divergence. A representation of the evolution of $W$ and Fst for different bidirectional $\mathrm{Cl}$ situations (no $\mathrm{Cl}\left(I_{A}=I_{B}=0\right),\left(I_{A}=0.5 ; I_{B}=0.75\right),\left(I_{A}=0.75 ; I_{B}=0.75\right)\left(I_{A}=I ; I_{B}=0.75\right)$ ) and migration rate $\mathrm{m}$. Other parameter values are $s_{i}=0.5, P_{S R}=0.75,=0, t=1, g=100, N=50$. Each graph represents each phenotype tested (Haplodiploids FM and MD, diploids and haploids). W is represented on the left and Fst on the right. The higher the value for each variable, the darker the box.

without any selection whereas in haplodiploids, it happened only for selection coefficients above 0.25 (Table 2 ). We also noticed that for $I_{A}=0.5, I_{B}=0.75$ and for $I_{A}=0.75$, $I_{B}=0.75$, increases in Fst were higher for the FM phenotype than for the MD phenotype. In addition, we found that for middle incompatibility rates $\left(I_{A}=0.5, I_{B}=0.75\right)$ diploids showed a greater increase in genetic divergence than haploids (Table 2). At $s_{i}=0.375$ diploids showed a significantly higher Fst with CI than without, whereas in haploids it was non significant. In fact, Fst was already high for haploids without CI (Fst $\in[0.209 ; 0.225]$ compared to Fst $\in[0.105 ; 0.115]$ for diploids) and Fst reached almost the same values in diploids and haploids in the case of bidirectional CI (e.g. for $I_{A}=1, I_{B}=0.75$, Fst $\in$
$[0.749 ; 0.770]$ for haploids and Fst $\in[0.763 ; 0.784]$ for diploids). Due to the lack of heterozygotes in the haploid system, the purging of disadvantageous alleles was higher and resulted in higher Fst values, a phenomenon characteristic of our semi-dominant locus selection model.

The effect of bidirectional CI on differentiation can be summarized as: $\mathrm{MD}<\mathrm{FM}<<$ haploids < diploids.

\section{Combined effect of sib-mating and bidirectional $\mathrm{Cl}$ on genetic differentiation (Figure 5)}

Firstly, for all values of , Fst and $W$ remained higher in the presence of bidirectional CI except for extreme sib-mating situations where they converged. Thus bidirectional CI 


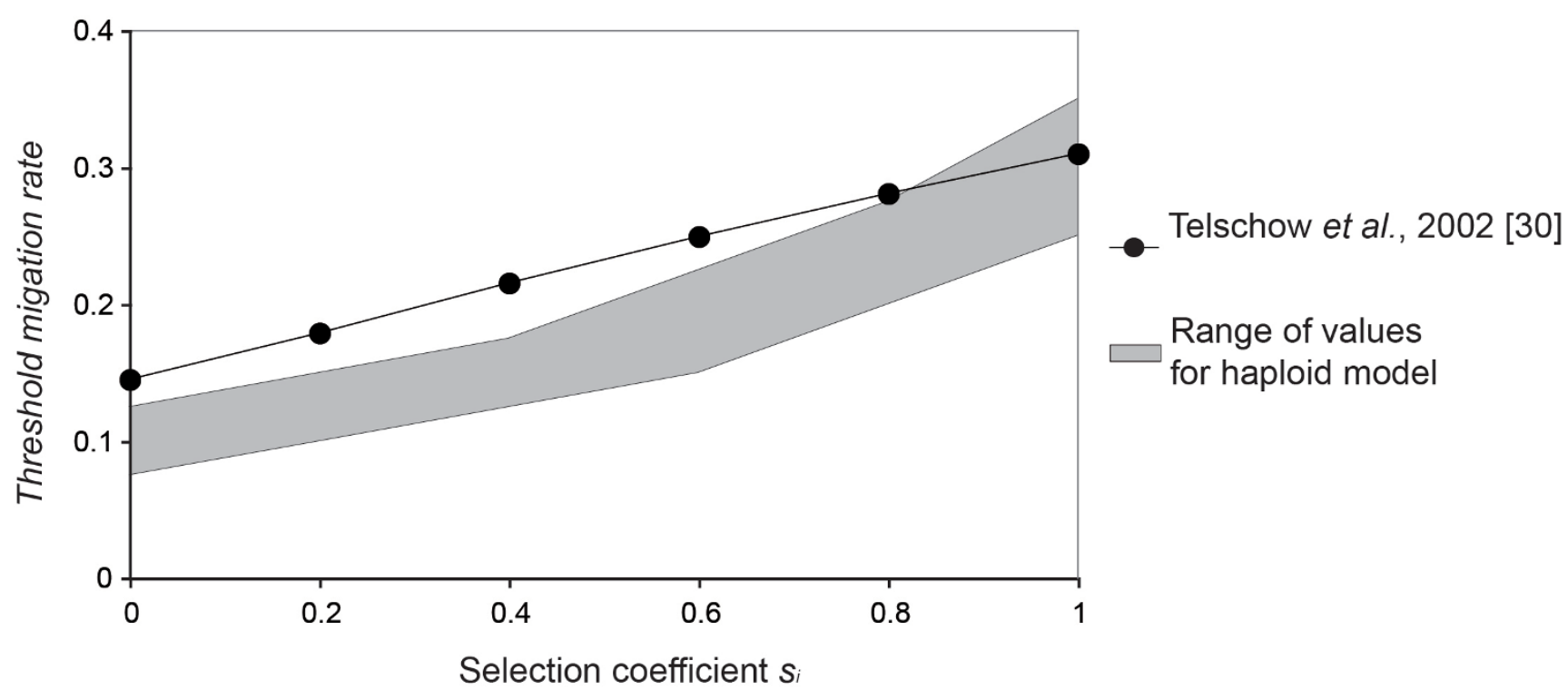

Figure 3

Comparison of the threshold migration rate in stochastic vs deterministic models. In the work by Telschow et al. [28], the threshold migration rate was defined as the migration rate beyond which Wolbachia diversity is no longer maintained. In our haploid model, a threshold migration range was represented as the migration rate valueswhere $W$ drops from 0.95 to 0.05 . The true threshold migration rate was assumed to be included in that range of migration rate values. Other parameter values were fixed at the same values as Telschow et al., $2002[28]: I_{A}=0.8, I_{B}=0.8, t=0.99, s r=0.5$.

enhanced genetic differentiation in all genetic systems from low to moderate levels of sib-mating.

Secondly, we observed that interaction between sib-mating and bidirectional $\mathrm{CI}$ for $W$ values depended on the genetic system being tested (Figure 5). In haplodiploids, sib-mating increased $W$ for middle bidirectional CI rate $\left(I_{A}=0.5 ; I_{B}=0.75\right)$ but had no effect for higher bidirectional CI rates. In diploids and haploids $W$ always showed a minimum at intermediate values of sib-mating. Indeed, it decreased with low levels of sib-mating and increased with high levels of sib-mating. The minimum value of $W$

Table 2: Test of the impact of bidirectional $\mathrm{Cl}$ on genetic divergence for different value of $s_{i}$

\begin{tabular}{|c|c|c|c|c|c|c|c|c|c|}
\hline Selection strength & 0 & 0.125 & 0.25 & 0.375 & 0.5 & 0.625 & 0.75 & 0.875 & 1 \\
\hline & Diplo & Diplo & Diplo & Diplo & Diplo & Diplo & Diplo & Diplo & Diplo \\
\hline$I_{A}=0.75$ & Haplo & Haplo & Haplo & Haplo & Haplo & Haplo & Haplo & Haplo & Haplo \\
\hline \multirow[t]{3}{*}{$I_{B}=I$} & & & HDFM & HDFM & HDFM & HDFM & HDFM & HDFM & HDFM \\
\hline & & & & HDMD & HDMD & HDMD & HDMD & HDMD & HDMD \\
\hline & Diplo & Diplo & Diplo & Diplo & Diplo & Diplo & Diplo & Diplo & Diplo \\
\hline$I_{A}=0.75$ & Haplo & Haplo & Haplo & Haplo & Haplo & Haplo & Haplo & Haplo & Haplo \\
\hline \multirow[t]{3}{*}{$I_{B}=0.75$} & & & HDFM & HDFM & HDFM & HDFM & HDFM & HDFM & HDFM \\
\hline & & & & HDMD & HDMD & HDMD & HDMD & HDMD & HDMD \\
\hline & & & & Diplo & Diplo & Diplo & Diplo & Diplo & Diplo \\
\hline$I_{A}=0.5$ & & & & & Haplo & Haplo & Haplo & Haplo & Haplo \\
\hline \multirow[t]{2}{*}{$I_{B} I_{B}=0.75$} & & & & & HDFM & HDFM & HDFM & HDFM & HDFM \\
\hline & & & & & & HDMD & HDMD & HDMD & HDMD \\
\hline
\end{tabular}

Each box is filled with the name of the system where bidirectional $\mathrm{Cl}$ significantly increased the Fst value on the selected locus (Mann-Whitney test, $p$-value $=0.05$ ).

Diplo $=$ Diploids, Haplo $=$ Haploids, HDFM $=$ Haplodiploid Female Mortality and HDMD $=$ Haplodiploid Male Development. Fixed parameters were set to $m=0.1, P_{S R}=0.75,=0, t=1, g=100, N=50$. 


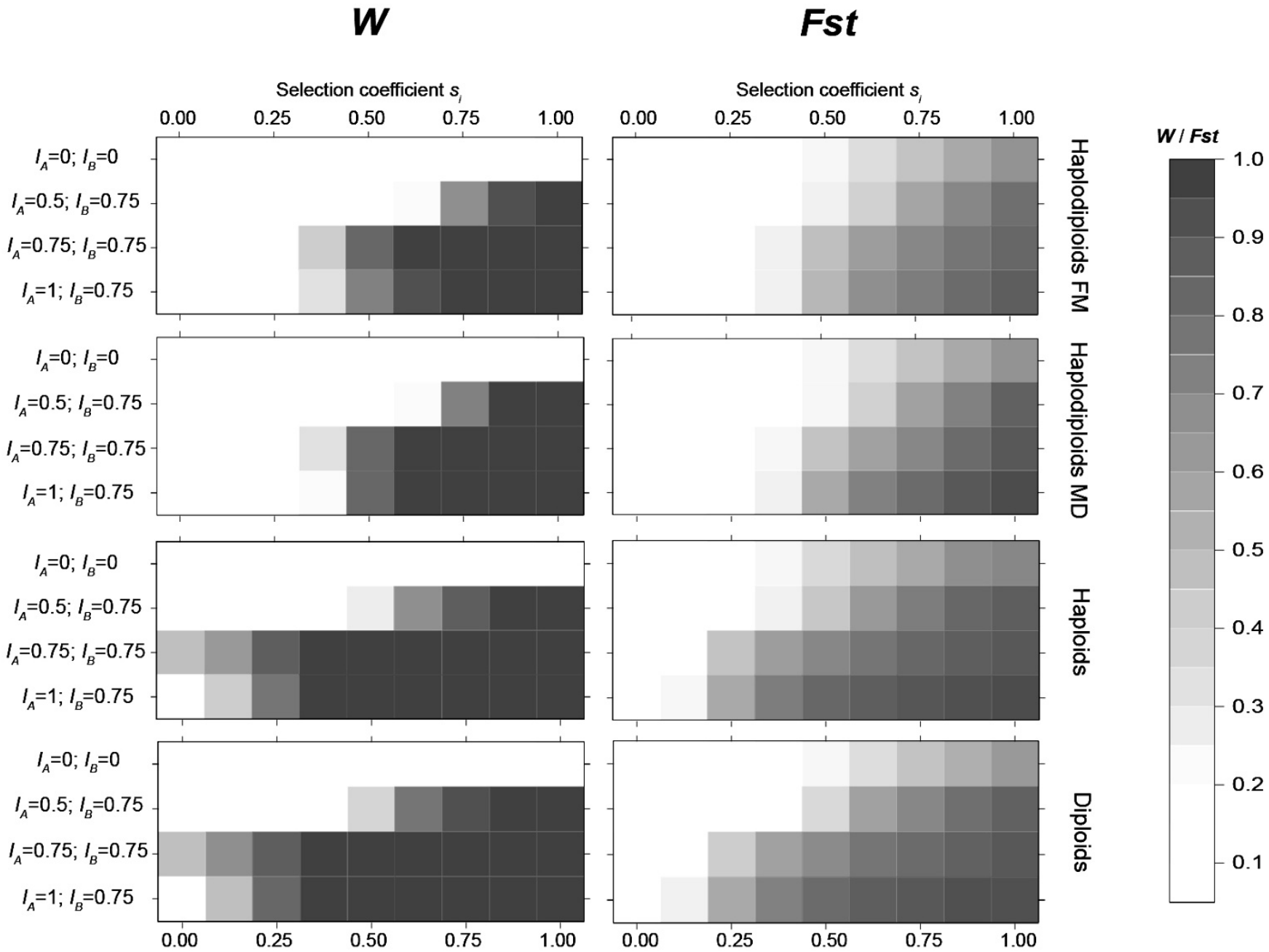

Figure 4

Effect of the selection coefficient $s_{i}$ on bidirectional $\mathbf{C l}$ and genetic divergence. A representation of the evolution of $W$ and Fst for different bidirectional $\mathrm{Cl}$ situations (no Cl $\left(I_{A}=I_{B}=0\right),\left(I_{A}=0.5 ; I_{B}=0.75\right),\left(I_{A}=0.75 ; I_{B}=0.75\right)\left(I_{A}=I ; I_{B}=0.75\right)$ ) and selection coefficients $s_{i}$. Other parameter values are fixed at $m=0 . I, P_{S R}=0.75,=0, t=1, g=100, N=50$. Each graph represents each phenotype tested (Haplodiploids FM and MD, diploids and haploids). W is represented on the left and Fst on the right. The higher the value for each variable, the darker the box.

was observed for $=0.25$ for middle bidirectional $\mathrm{CI}$ rates $\left(I_{A}=0.5 ; I_{B}=0.75\right)$ and for $=0.875$ for higher bidirectional CI rates.

In haplodiploids (Figure 5), sib-mating increased Fst while in diploids and haploids, this phenomenon was only observed from middle bidirectional CI $\left(I_{A}=0.5 ; I_{B}=\right.$ 0.75 ) to no bidirectional CI.

\section{Effect of transmission efficiency}

We also tested the effect of a reduction in transmission efficiency $t$ on the different genetic systems. We first observed that $W$ decreased for higher values of $t$ in haplodiploids compared to the other genetic systems. In the haplodiploid genetic system, maintenance of Wolbachia diversity was observed only for values of $t$ equal to or above 0.95. For the other genetic systems, maintenance was as low as 0.90 . Therefore, the diversity of Wolbachia strains can only be maintained in host populations in which transmission efficiency is almost complete, especially in haplodiploid systems.

\section{Sensitivity of stochastic model to generation length and population size}

First, we tested the effect of various population sizes on the results $(N=50,200,500,1000)$. Values of the variables Fst and $W$ were found to be stable for population size above $N=50$. We saw a decrease in the standard error of 


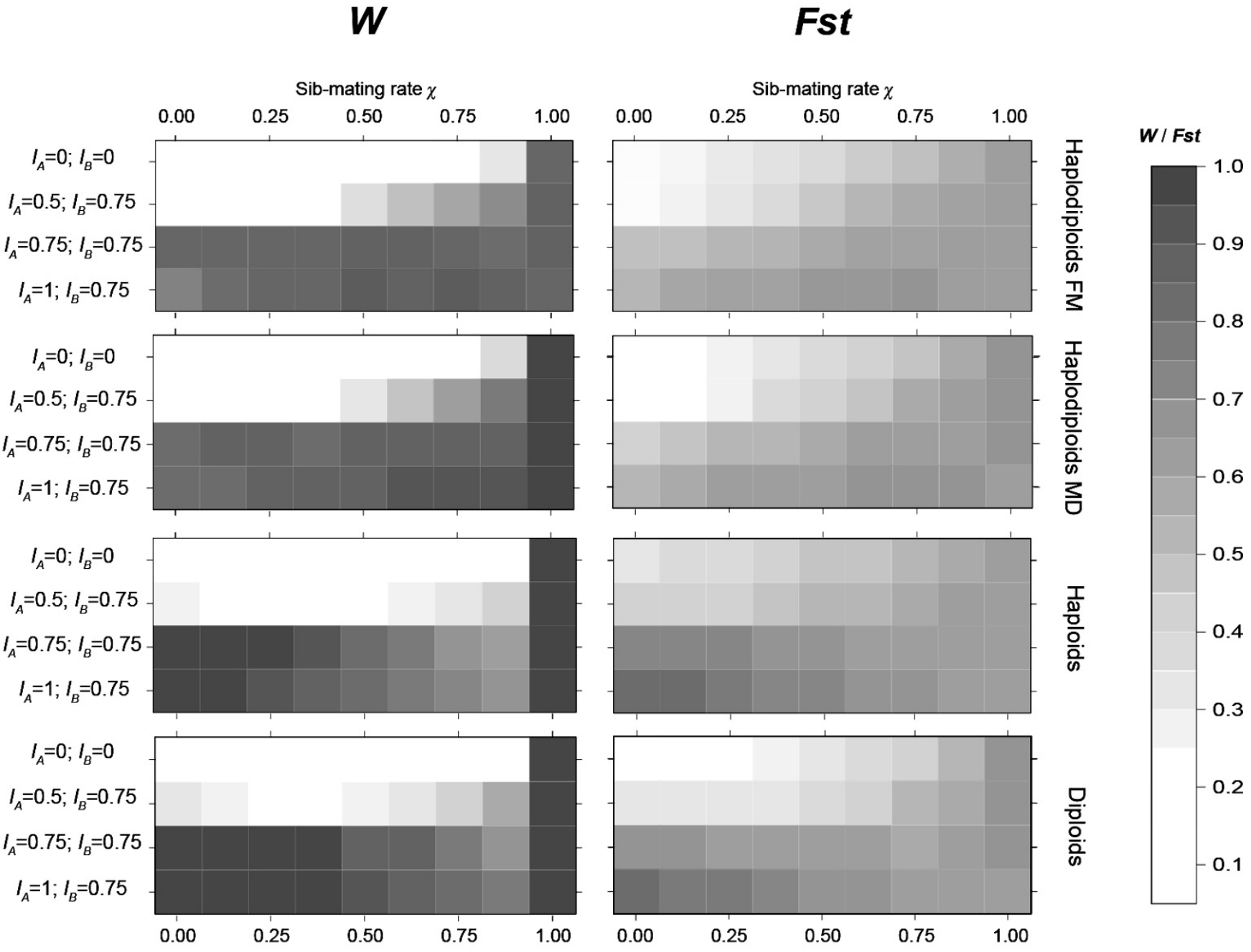

Figure 5

Effect of the sib-mating rate $\chi$ on bidirectional $\mathbf{C l}$ and genetic divergence. $A$ representation of the evolution of $W$ and Fst for different bidirectional $\mathrm{Cl}$ situations (no Cl $\left.\left(I_{A}=I_{B}=0\right),\left(I_{A}=0.5 ; I_{B}=0.75\right),\left(I_{A}=0.75 ; I_{B}=0.75\right)\left(I_{A}=I ; I_{B}=0.75\right)\right)$ and for different values of sib-mating rate. Other parameter values are fixed at $m=0.1, s_{i}=0.5, P_{S R}=0.75, t=I, g=100, N=50$. Each graph represents each phenotype tested (Haplodiploids FM and MD, diploids and haploids). W is represented on the left and Fst on the right. The higher the value for each variable, the darker the box.

the variables estimation as population size increased because of reduction of genetic drift effect. Variables $W$ and Fst were found to be slightly lower for population size $N=50$ but not significantly so.

Second, we increased generation length to see long-term dynamics of the variables $W$ and Fst. We found that if $W$ was close to 1 or equal to 0 , it was stable after 100 generations for long time ( $>2000$ generations). For intermediate values recorded at $g=100, W$ tended to drop to 0 after a thousand generations. We noted that the observed number of generations before reaching 0 were lower for the MD phenotype than for FM phenotype. We observed also that if we increased population size, we augmented the number of generations necessary for $W$ to reach zero. For Fst, we observed the same dynamic as $W$, following the decreasing influence of bidirectional CI when $W$ decreased.

\section{Discussion}

\section{Convergence of deterministic and stochastic models}

In accordance with previously published models of haploids [28], we first observed that below a threshold migration rate, Wolbachia strains were maintained. However our values for the threshold migration rate were lower than the ones observed in the deterministic model of Telschow 
et al. [28] because our approach relies on a stochastic modeling, with a high impact of genetic drift. Therefore it is possible for a Wolbachia strain to be lost due to genetic drift if it reaches a low frequency. This was not possible in previous models. We also observed that the threshold migration rates for both the stochastic and previous deterministic approach became closer at higher selection rates. This was due to drift effects on stochastic models that became negligible in comparison to selection at very high values of $s_{i}$.

We also saw that the $W$ variable took a value of 0 or near 1 if the program was run a long duration. This meant that either the Wolbachia strain diversity was maintained in almost all repetitions or that one strain invaded both insect populations. It reflects a dynamic where there are two stable equilibria (1) one Wolbachia strain in the two populations or (2) two strains present but each strain is restricted to a different population. In this respect, our results are similar to those of other determinist approaches, which have investigated the dynamics of strains harbored by different populations [28,31].

Finally, the only previous model that has considered sibmating found that Wolbachia is harder to spread in populations submitted to sib-mating [39]. It even cannot persist beyond a threshold level of sib-mating because CI expression becomes too rare. An analogous result in our model would have been the invasion of one strain of Wolbachia in to both insect populations i.e. $W=0$. But in fact in our model, selection allows Wolbachia to be maintained at very high values because when the sib-mating rate is high, Wolbachia and the nuclear locus under selection segregate together. However, at near complete sibmating, it was possible for Wolbachia strains to be maintained only if transmission efficiency was total or neartotal.

\section{Specific responses of haplodiploid populations under bidirectional $\mathrm{Cl}$}

Our model highlights that bidirectional $\mathrm{CI}$ in the haplodiploid genetic system is less likely to maintain Wolbachia diversity as well as enhance genetic differentiation between differentially infected populations. The possibility of the production of arrhenotokous male following migration in the first generation may explain the lower threshold migration rate within haplodiploid systems. Differentiation in all systems is maintained because first migrant females are more likely to cross with an individual harboring a different Wolbachia strain and, hence, more likely to suffer more from CI than a resident female. In haplodiploid genetic systems however, CI produces as many males (FM) or even more males (MD) than in compatible crosses. These males harbor Wolbachia from the immigrant population. Therefore, the relative frequency of males harboring immigrant Wolbachia is higher in the following generation in haplodiploids than in haploids and diploids. As a result, in the second generation, recently migrated lineages have more available males having the same Wolbachia and, therefore, suffer less from CI effect than those of the first generation. These factors facilitate invasion of the immigrant bacteria strain and Wolbachia diversity is consequently harder to maintain for haplodiploids.

In parallel, we note that there is a high sensitivity to transmission rate variation in the haplodiploid systems. Within the literature, there are records of transmission rates that exceed 95\% (for example 96-97\% in Culex pipiens [45] or 98.6\% in Drosophila simulans [46]). Our work has shown that such variations at high rates have a weak impact on Wolbachia dynamics in haploid and diploid genetic systems, but a high impact in haplodiploid genetic systems.

As shown in previous models, when Wolbachia diversity is maintained, Fst increases because bidirectional CI results in migrant offspring death and, hence, in a reduction of the effective migration rate $[28,30]$. We observed that the effect on genetic divergence was weaker in haplodiploids than in other systems, even when both Wolbachia strains were maintained. As for Wolbachia, this observation appears to arise from the production of males in incompatible crosses in haplodiploids, allowing more introgression of the immigrant allele to occur.

\section{Sib-mating compensates for differences between haplodiploids and other systems}

Very high values of sib-mating always favor divergence and maintenance of Wolbachia infection diversity by maintaining the association between Wolbachia and nuclear genes. But at lower values of sib-mating, in haploid and diploid systems, we observed a reduced $W$ in the presence of sib-mating. This reduction of $W$ due to sibmating did not occur in haplodiploid systems. However, in haplodiploid systems, sib-mating inhibits the introgression associated with haploid male production. In all systems, when individuals mate with sibs, incompatible crosses occur less frequently and, hence, reduce the impact of bidirectional CI on genetic divergence. But in the case of haplodiploids, as discussed previously, a reduction of $W$ in the absence of sib-mating, relies on the production of males from incompatible crosses. These males, in effect, reduce the fitness of local females (i.e. local Wolbachia) by causing $\mathrm{CI}$, and, hence favoring the introgression of exotic Wolbachia strain. In this situation, sib-mating prevents introgression by affording sibling males a higher reproductive success in comparison to non-sibling males. Thus overall, the effect of sib-mating on $W$ is less pronounced in haplodiploids than in haploids or diploids. 


\section{The implications of bidirectional $\mathrm{Cl}$ on speciation by reinforcement}

The present model shows that sib-mating, which is frequently observed in gregarious haplodiploid parasitoids, can complement less efficient bidirectional CI. In this study, we have ascertained that sib-mating is capable of enhancing the weak effect of bidirectional CI in haplodiploid systems. Moreover, in a previous work on unidirectional CI in haplodiploids, sib-mating was shown to reduce the loss of population growth rate via CI during Wolbachia invasion [40]. This contribution of sib-mating in the Wolbachia-induced differentiation will have an effect on the next step of speciation according to the reinforcement theory. Reinforcement speciation theory predicts that postmating isolation mechanisms, if maintained through generations, will be replaced by less costly premating isolation mechanisms [47]. In the case of postmating isolation induced by bidirectional CI, a previous model has shown that premating isolation mechanisms are likely to be selected for [30]. Nevertheless, evolution of premating isolation (further steps of reinforcement) may be counteracted by sib-mating because sib-mating may limit the cost of Wolbachia postmating mechanisms. We therefore expect selection for premating isolation mechanisms to be weaker in haplodiploids encountering frequent sib-mating. Interestingly, in haplodiploid spider mites, it has been demonstrated that Wolbachia induces sib-mating in infected individuals [48]. The present model suggests that such induced sib-mating may be an efficient alternative strategy to CI for maintaining Wolbachia in a population. In addition, with MD and FM, the other effect of Wolbachia in haplodiploids is thelytokous parthenogenesis induction (PI). It can be viewed as the extreme inbreeding strategy and an efficient strategy for Wolbachia to maintain strain diversity jointly with local adaptation of its host in parapatric populations. In conclusion, we emphasize that ability for Wolbachia to maintain postmating isolation and select for premating isolation mechanisms is dependent on the mating system of the host species. Inbreeding mating systems should be further investigated in future work on Wolbachia.

\section{Reconsidering the Nasonia case}

The clearest example of speciation likely to have been induced by Wolbachia is "the Nasonia case". The three species of the Nasonia complex, are bidirectionally incompatible, due to either MD or FM [23] and are genetically differentiated [49]. The hypothesis is that bidirectional CI is the major agent responsible for their primary isolation $[22,23]$. Reinforcement has not occurred totally yet since no premating isolation had arisen. The closest related species, Nasonia giraulti and N. longicornis occur in allopatry, which may explain the absence of premating isolation. However, these two species can occur in sympatry with the more distantly related species $N$. vitripennis but no pre- mating isolation mechanisms have evolved. We can speculate in light of our results as to what the predominant role of bidirectional CI versus the important contribution of sib-mating in Nasonia speciation is by considering that:

- in haplodiploids, Wolbachia induced bidirectional CI alone is less efficient to contribute to genetic divergence.

-Nasonia species are gregarious and exhibit sib-mating behavior, and even within host mating, when females emerged already mated from the host puparium [50].

Our hypothesis is that bidirectional CI and sib-mating have in combination contributed to genetic divergence of Nasonia species and that this has occurred in a framework in which frequent sib-mating has prevented evolution of premating isolation mechanisms.

\section{Implications for biological control strategies using parasitoid agents}

CI inducing Wolbachia have already been used as a biological control strategy to control insect pest growth rate by introducing an infected conspecific [51,52]. Biological control programs that utilize endemic wasp species can conceivably make use of the knowledge that Wolbachia could play a role in local adaptation. Introduced biological control agents often interact with local populations that are specialized on a different host, or can be composed of different genotypes reflecting virulence on different hosts $[53,54]$. Given that sib-mating coupled with bidirectional $\mathrm{CI}$ contribute to maintaining host specialization, it may be envisaged to introduce agents with different host niche and infected with different Wolbachia strains. If bacteria strains are bidirectionally incompatible, then reproductive isolation will occur. On the one hand, too many incompatible crosses can render bidirectional CI deleterious due to the reduction in population growth associated with it. On the other hand, a sib-mating process can compensate for these same detrimental effects. Local adaptation is, therefore, reinforced over generations so that mixing with locally avirulent populations is avoided. Existing biological control strategies may offer the opportunity to field-test the scenarios developed in this model.

\section{Conclusion}

Our results show that the implications of Wolbachia in genetic differentiation depend on crucial interaction between biological traits such as the genetic system and sib-mating rate. Complex analysis of these factors reveals a general trend that permits two principal conclusions to be drawn. Firstly, in haplodiploid systems bidirectional CI possesses a limited ability to maintain Wolbachia diversity and, by extrapolation, a limited ability to maintain genetic divergence of locally adapted genes. Secondly, sib- 
mating behavior is particularly efficient in haplodiploid systems to counteract a reduced impact of bidirectional CI on genetic divergence. However, it may slow down the speciation process by reducing the postmating isolation cost of Wolbachia and, thereby, the selection for premating isolation. Therefore, speciation induced by bidirectional CI alone is expected to occur less frequently and for a narrower range of parameters in haplodiploids compared to diploids.

\section{Authors' contributions}

All the authors, AB, SD, FV and JFS, participated in the design of the study and drafted the article. In addition, $\mathrm{AB}$ wrote the R source code with the CIParasitoid package and analyzed the output data. SD also contributed to the development of the R source code.

\section{Additional material}

\section{Additional file 1}

$R$ package CIParasitoid for Windows XP. Package CIParasitoid for $R$ containing the program presented here. It has been built on $R$ 2.8.0 for Windows XP. The latest version of $R$ along with installation instructions can be found at http://www.r-project.org/.

Click here for file

[http://www.biomedcentral.com/content/supplementary/14712148-9-185-S1.zip]

\section{Additional file 2}

$R$ package CIParasitoid for Mac OS X Intel processor. Package CIParasitoid for $R$ containing the program presented here. It has been built on $R$ 2.8.0 for MacOSX Intel processor. The latest version of $R$ along with installation instructions can be found at http://www.r-project.org/. First extract the tar.gz binary file from the .zip file before any installation in $R$. Click here for file

[http://www.biomedcentral.com/content/supplementary/14712148-9-185-S2.zip]

\section{Additional file 3}

$R$ package CIParasitoid for Linux. Package CIParasitoid for R containing the program presented here. It has been built on R 2.8.0 for Linux. The latest version of $R$ along with installation instructions can be found at http://www.r-project.org/. First extract the tar.gz binary file from the .zip file before any installation in $R$.

Click here for file

[http://www.biomedcentral.com/content/supplementary/14712148-9-185-S3.zip]

\section{Additional file 4}

$R$ package CIParasitoid for Mac OS X PowerPC processor. Package CIParasitoid for $R$ containing the program presented here. It has been built on R 2.8.0 for MacOSX PowerPC processor. The latest version of $R$ along with installation instructions can be found at http://www.rproject.org/. First extract the tar.gz binary file from the .zip file before any installation in $R$.

Click here for file

[http://www.biomedcentral.com/content/supplementary/14712148-9-185-S4.zip]

\section{Acknowledgements}

This work was support by Research Unit IRD UR072. We want to acknowledge Michelle Schiffer for English correction and useful comments. We also thank Laure Kaiser-Arnauld, Olivier Dangles, Pascal Campagne and two anonymous reviewers for their useful comments.

\section{References}

I. Perlman SJ, Hunter MS, Zchori-Fein E: The emerging diversity of Rickettsia. Proceedings of the Royal Society B: Biological Sciences 2006, 273( ( 598):2097-2106.

2. Laven H: Crossing Experiments with Culex Strains. Evolution 195I, 5(4):370-375.

3. Stouthamer R, Luck RF, Hamilton WD: Antibiotics cause parthenogenetic Trichogramma (Hymenoptera:Trichogrammatidae) to revert to sex. Proceedings of the National Academy of Sciences 1990, 87(7):2424-2427.

4. Montenegro H, Solferini VN, Klaczko LB, Hurst GDD: Male-killing Spiroplasma naturally infecting Drosophila melanogaster. Insect Molecular Biology 2005, I 4(3):28I-287.

5. Zchori-Fein E, Gottlieb Y, Kelly SE, Brown JK, Wilson JM, Karr TL, Hunter MS: A newly discovered bacterium associated with parthenogenesis and a change in host selection behavior in parasitoid wasps. Proceedings of the National Academy of Sciences 200I, 98(22): I2555-12560.

6. Moran NA, Russell JA, Koga R, Fukatsu T: Evolutionary relationships of three new species of Enterobacteriaceae living as symbionts of aphids and other insects. Applied and Environmental Microbiology 2005, 7 I (6):3302-33 I0.

7. Rousset F, Bouchon D, Pintureau B, Juchault P, Solignac M: Wolbachia endosymbionts responsible for various alterations of sexuality in arthropods. Proceedings of the Royal Society B: Biological Sciences 1992, 250(1328):91-98.

8. Werren JH: Biology of Wolbachia. Annual Review of Entomology 1997, 42:587-609.

9. Stouthamer R, Breeuwer JA, Hurst GD: Wolbachia pipientis: microbial manipulator of arthropod reproduction. Annual Review of Microbiology 1999, 53:71-102.

10. Ghelelovitch S: Sur le determinisme genetique de la sterilite dans le croisement entre differentes souches de Culex autogenicus Roubaud. Comptes Rendus de l'Academie des Sciences 1952, 24:2386-2388.

II. Reed KM, Werren JH: Induction of paternal genome loss by the paternal-sex-ratio chromosome and cytoplasmic incompatibility bacteria (Wolbachia): a comparative study of early embryonic events. Molecular Reproduction and Development 1995, 40(4):408-4I8.

12. O'Neill SL, Karr TL: Bidirectional incompatibility between conspecific populations of Drosophila simulans. Nature 1990, 348(6297): $178-180$.

13. Breeuwer JAJ: Wolbachia and cytoplasmic incompatibility in the spider mites Tetranychus urticae and $T$. turkestani. Heredity 1997, 79(I):4I-47.

14. Vavre F, Fleury F, Varaldi J, Fouillet P, Bouletreau M: Evidence for female mortality in Wolbachia-mediated cytoplasmic incompatibility in haplodiploid insects: epidemiologic and evolutionary consequences. Evolution 2000, 54(1): $191-200$.

15. Breeuwer JA, Werren JH: Microorganisms associated with chromosome destruction and reproductive isolation between two insect species. Nature 1990, 346(6284):558-560.

16. Turelli M, Hoffmann AA: Rapid spread of an inherited incompatibility factor in California Drosophila. Nature 1991, 353(6343):440-442.

17. Rokas II: Wolbachia as a speciation agent. Trends in Ecology and Evolution 2000, I5(2):44-45.

18. Wade MJ: Infectious speciation. Nature 200I, 409(682I):675-677.

19. Werren JH: Wolbachia and speciation. In Endless Forms: Species and Speciation New York, NY: Oxford University Press, USA; 1998:245-260.

20. Zimmer C: Wolbachia. A tale of sex and survival. Science 200I, 292(55 19): 1093-1095.

21. Hilgenboecker K, Hammerstein P, Schlattmann P, Telschow A, Werren JH: How many species are infected with Wolbachia? - a statistical analysis of current data. FEMS Microbiology Letters 2008, $28 I(2): 215-220$. 
22. Bordenstein SR, O'Hara FP, Werren JH: Wolbachia-induced incompatibility precedes other hybrid incompatibilities in Nasonia. Nature 200I, 409(682I):707-7I0.

23. Bordenstein SR, Werren JH: Bidirectional incompatibility among divergent Wolbachia and incompatibility level differences among closely related Wolbachia in Nasonia. Heredity 2007, 99(3):278-287.

24. Weeks AR, Tracy Reynolds K, Hoffmann AA: Wolbachia dynamics and host effects: what has (and has not) been demonstrated? Trends in Ecology and Evolution 2002, I 7(6):257-262.

25. Coyne JA, Orr HA: Speciation. Sinauer Associates Sunderland, MA; 2004.

26. Ballard JW, Chernoff B, James AC: Divergence of mitochondrial DNA is not corroborated by nuclear DNA, morphology, or behavior in Drosophila simulans. Evolution 2002, 56(3):527-545.

27. Jaenike J, Dyer KA, Cornish C, Minhas MS: Asymmetrical reinforcement and Wolbachia infection in Drosophila. PLoS Biology 2006, 4(I 0): e325.

28. Telschow A, Hammerstein P, Werren JH: The effect of Wolbachia on genetic divergence between populations: models with two way migration. The American Naturalist 2002, 160:S54-S66.

29. Telschow A, Hammerstein P, Werren JH: Effects of Wolbachia on Genetic Divergence Between Populations: Mainland-Island Model I. Integrative and Comparative Biology 2002, 42(2):340-35I

30. Telschow A, Hammerstein P, Werren JH: The effect of Wolbachia versus genetic incompatibilities on reinforcement and speciation. Evolution 2005, 59(8):1607-1619.

31. Telschow A, Yamamura N, Werren JH: Bidirectional cytoplasmic incompatibility and the stable coexistence of two Wolbachia strains in parapatric host populations. Journal of Theoretical Biology 2005, 235(2):265-274.

32. Egas $M$, Vala $F$, Breeuwer JA: On the evolution of cytoplasmic incompatibility in haplodiploid species. Evolution 2002 56(6): I I0I-I 109

33. Dubuffet A, Alvarez CIR, Drezen JM, van Alphen JJM, Poirie M: Do parasitoid preferences for different host species match virulence? Physiological Entomology 2006, 3 I(2): I70-I77.

34. Li Y, Lu JF, Feng CJ, Ke XIN, Fu W: Role of venom and ovarian proteins in immune suppression of Ostrinia furnacalis (Lepidoptera: Pyralidae) larvae parasitized by Macrocentrus cingulum (Hymenoptera: Braconidae), a polyembryonic parasitoid. Insect Science 2007, I4(2):93-100.

35. Webb BA, Strand MR, Dickey SE, Beck MH, Hilgarth RS, Barney WE, Kadash K, Kroemer JA, Lindstrom KG, Rattanadechakul W, et al.: Polydnavirus genomes reflect their dual roles as mutualists and pathogens. Virology 2006, 347(I): I60-I74.

36. Godfray HC): Parasitoids: Behavioral and Evolutionary Ecology. Princeton, NJ: Princeton University Press; 1994.

37. Godfray HCJ, Cook JM: Mating systems of parasitoid wasps. Cambridge: Cambridge University Press; 1997.

38. Hardy ICW: Sex ratio and mating structure in the parasitoid Hymenoptera. Oikos 1994, 69(I):3-20.

39. Engelstadter J, Charlat S, Pomiankowski A, Hurst GD: The evolution of cytoplasmic incompatibility types: integrating segregation, inbreeding and outbreeding. Genetics 2006 I 72(4):260I-26II.

40. Branca A, Dupas S: A model for the study of Wolbachia pipientis Hertig (Rickettsiales: Rickettsiaceae)-induced cytoplasmic incompatibility in arrhenotokous haplodiploid populations: Consequences for biological control. Annales de la Societe entomologique de France 2006, 42(3-4):443-448.

4I. Jansen VA, Turelli M, Godfray HC: Stochastic spread of Wolbachia. Proceedings of the Royal Society B: Biological Sciences 2008 275( ( 652):2769-2776.

42. Antolin MF: A genetic perspective on mating systems and sex ratios of parasitoid wasps. Researches on Population Ecology 1999 4I(I):29-37.

43. Hamilton WD: Extraordinary sex ratios. A sex-ratio theory for sex linkage and inbreeding has new implications in cytogenetics and entomology. Science 1967, I 56(774):477-488.

44. Team RDC: R: A language and environment for statistical computing. Vienna, Austria: R Foundation for Statistical Computing; 2004.

45. Rasgon JL, Scott TW: Wolbachia and cytoplasmic incompatibility in the California Culex pipiens mosquito species complex: parameter estimates and infection dynamics in natural populations. Genetics 2003, I 65(4):2029-2038.

46. Turelli M, Hoffmann AA: Cytoplasmic incompatibility in Drosophila simulans: dynamics and parameter estimates from natural populations. Genetics 1995, I40(4): I319-1338.

47. Dobzhansky T: Genetics and the Origin of Species. New York, NY: Columbia University Press; 1937.

48. Vala F, Egas M, Breeuwer JA, Sabelis MW: Wolbachia affects oviposition and mating behaviour of its spider mite host. Journal of Evolutionary Biology 2004, I 7(3):692-700.

49. Campbell BC, Steffen-Campbell JD, Werren JH: Phylogeny of the Nasonia species complex (Hymenoptera: Pteromalidae) inferred from an internal transcribed spacer (ITS2) and 285 rDNA sequences. Insect Molecular Biology 1993, 2(4):225-237.

50. Drapeau MD, Werren JH: Differences in mating behaviour and sex ratio between three sibling species of Nasonia. Evolutionary Ecology Research 1999, I(2):223-234.

5I. Douglas AE: Symbiotic microorganisms: untapped resources for insect pest control. Trends in Biotechnology 2007, 25(8):338-342.

52. Zabalou S, Riegler M, Theodorakopoulou M, Stauffer C, Savakis C, Bourtzis K: Wolbachia-induced cytoplasmic incompatibility as a means for insect pest population control. Proceedings of the National Academy of Science 2004, I 0 I (42): I 5042-I 5045.

53. Ngi-Song AJ, Overholt WA, Stouthamer R: Suitability of Busseola fusca and Sesamia calamistis (Lepidoptera: Noctuidae) for the development of two populations of Cotesia sesamiae (Hymenoptera: Braconidae) in Kenya. Biological Control 1998 , I 2(3):208-2 I4.

54. Gitau CW, Gundersen-Rindal D, Pedroni M, Mbugi PJ, Dupas S: Differential expression of the CrVI haemocyte inactivationassociated polydnavirus gene in the African maize stem borer Busseola fusca (Fuller) parasitized by two biotypes of the endoparasitoid Cotesia sesamiae (Cameron). Journal of Insect Physiology 2007, 53(7):676-684.
Publish with Biomed Central and every scientist can read your work free of charge

"BioMed Central will be the most significant development for disseminating the results of biomedical research in our lifetime. "

Sir Paul Nurse, Cancer Research UK

Your research papers will be:

- available free of charge to the entire biomedical community

- peer reviewed and published immediately upon acceptance

- cited in PubMed and archived on PubMed Central

- yours - you keep the copyright
BiolMedcentral 